\title{
GEOMETRIC ANALYSIS OF A SPACE GRID STRUCTURE BY AN INTEGRATED 3D SURVEY APPROACH
}

\author{
Michele Russo $^{1}$, Valentina Russo ${ }^{2}$ \\ ${ }^{1}$ History, Representation and Restoration of Architecture Dept., Sapienza University in Rome, m.russo@uniroma1.it \\ ${ }^{2}$ Errealcubo Studio, ing.valentinarusso@gmail.com
}

\section{Commission II}

KEY WORDS: multi-source 3D data, space grid structures, geometrical analysis, as-built assessment, 3D reconstruction

\begin{abstract}
:
The survey and modeling of spatial grid structures is often a challenging task to solve, due to the many bottlenecks present in the reverse modelling process. Geometrical and material complexity, scale variation, shadows effects, low accessibility, mismatch between similar elements, metrical validation, data management are all critical passages. They must be solved to obtain a reliable data able to solve the system maintenance and intervention requirements. In these cases, in particular, when the structure is difficult to be accessed, it is essential to plan an integrated survey approach. This paper shows how the survey and modelling of an impressive structure was carried out using both TLS and RPAS photogrammetry. After being validated, the data is integrated into a single point cloud that completely describes the structure, eliminating shadow areas and hidden parts. In the modeling process, particular attention was paid to verifying the correspondence between as-built and project. Finally, an element of particular interest is represented by the attempt to recognize the individual construction components belonging to specified geometric and dimensional classes, with the advantage of obtaining a complete model that is as faithful as possible to the real situation of the structure.
\end{abstract}

\section{INTRODUCTION}

The integration between 3D surveying techniques allows gaining deep knowledge in multiple domains. The last decade's research showed its crucial role in understanding complex architectures, exploiting the potential of each survey methodology, optimizing on-site presence, limiting shadow areas, and increasing data redundancy and reliability. This latter represents a crucial passage in converting parametric 3D models, tracing the reverse modeling accuracy.

Some of the first examples in the architecture domain faced the problem of merging image-based with range-based data, creating multi-resolution models (Guidi et al., 2009). After more than one decade, integrated surveys use multiple sensors and orientation algorithms (Adamopoulos and Rinaudo, 2019), involving interoperable platforms to manage 3D data, parametric modeling, and the Scan-to-BIM process (Lo Brutto et al., 2021). Besides, the possibility to connect directly $2 \mathrm{D}$ data with $3 \mathrm{D}$ space introduce new survey paradigms and instruments for real-tovirtual convergence (Abergel et al., 2021).

In this scenario, it seems that it is possible to survey any architecture, regardless of the shape complexity, spaces articulation, or object scale. However, this description does not suit reality. There are still many challenging architectonic examples that propose complex working conditions, free-form shapes, narrow or articulated spaces, and optically noncooperative materials. Indeed, the 3D data integration is helpful in specific complex case studies in which redundancy, shadows effects, and validation have a primary role in the process. It also allows planning a custom acquisition approach to answer specific operating conditions or objects/environments features (Perfetti et al., 2018).

An integrated survey approach may be a necessary but nonsufficient condition to represent complex architectures, which require more effort in the data process, interpretation, and modeling activities. That is the case of a little-investigated domain presented in the paper, the space grid architectonic structures (Chilton, 2000). They belong to a specific class of elements that come back from the cast-iron buildings of the late 18th century, covering different functions (bridge, towers, roof). The high formal complexity of these elements and their diffusion, often limited to the large architectures, makes this typology still little studied (Figure 1). Nevertheless, it is crucial to search for new geometrical and material analysis methods, compare the differences between the project and the as-built, monitor the structure behavior, and plan techno structural interventions for the future. The research suggests an integrated 3D survey and modeling process to solve many described bottlenecks for these particular structures.

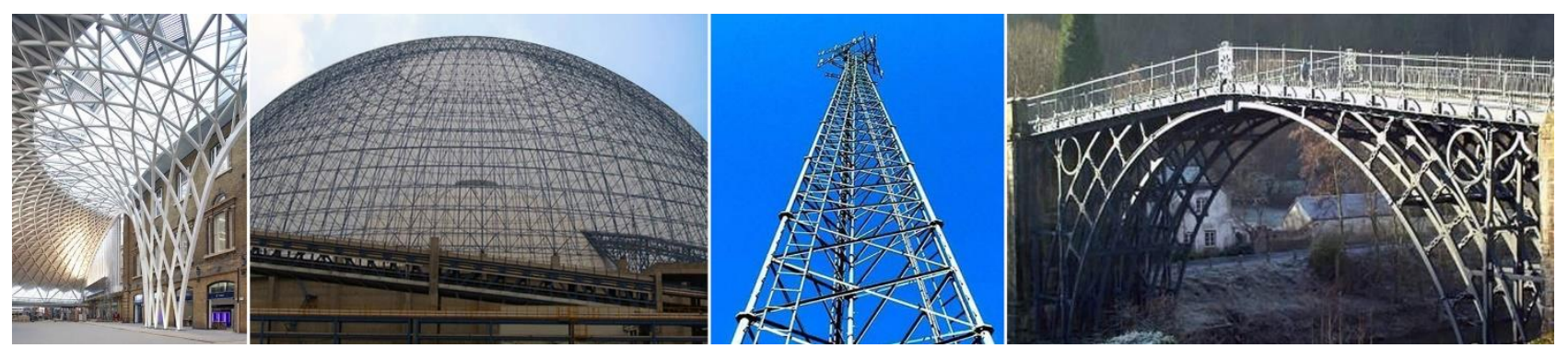

Figure 1: Different architectural types based on space grid structures. 


\section{RELATED WORKS}

The topic of grid structures involves different research areas, mainly referred to constructions or existing domain (Figure 1). Most recent advances focus on element construction, strain analysis, and structural simulation. On the contrary, few references are present about geometrical acquisition and modeling of the structures (Wei et al., 2021) or their connection components (Pereira et al., 2021). This lack is probably due to the high topological complexity of the artifacts and the challenging attempt to obtain reliable 3D data. The components' spatial distribution, accessibility, dimension, transparent doubleside cover, variation in detail, color, and material (Candela et al., 2019) increase the system complexity. In addition, both the shadow areas generated by nodes and mullions and the geometrical similarities between elements prevent correct 3D data recognition. The survey methods, data interpretation, and 3D modeling are central in our research.

The external survey conditions combined with the particular characteristics of the structure affect all reverse modelling phases. Most of the research starts with a priori assumptions that mitigate this complexity. The global structure can be considered as a simple wireframe (Liu et al., 2017), composed of elements that are assumed as linear (Bacharidis et al., 2020), assigning to the mullions a tubular shape [Martin et al., 2014). Huang et al. (Huang et al. 2013) suggest a features extraction approach to create a curved architectonic skeleton from an unstructured 3D point cloud, providing a simplified structure representation. Morioka et al. (Morioka et al. 2013) apply Delaunay's algorithm on the raw 3D point cloud, extracting a simplified graph representing the structure's topology. For the single components of the grid structure, they propose an optimized construction process based on cylindrical surfaces centered along the edges of the graph, improving the accuracy in the reconstruction of the parametric geometries. Finally, Su et al. (Su et al., 2018) employ an optical-based method, using 3D architectural networks to analyze the existing topology at a micrometric resolution. The method is based on an automated acquisition and segmentation process, obtaining high-resolution 2D images of each crosssection of the structure. Image processing algorithms provide a model containing reliable structural and topological features, predicting the mechanical response, as presented by Liu et al. (Liu et al., 2019) using image-based 3D techniques.

All these examples foresee a problem simplification. An integrated 3D survey approach can supply optimized and flexible procedures, redundant data systems, and analysis for accuracy validation. High accuracy in the survey and modeling process can lead to a dense and reliable grid components acquisition, opening several automatic recognition and parameterization scenarios (Yang et al., 2020).

In the 3D survey and modeling field, several bottlenecks may affect the acquisition and the construction of the digital parametric structure concerning the 3D acquisition methodology. In the case of active and passive systems, it is essential to use UAVs equipped with different instruments (RGB cameras, Lidar, IR cameras) that integrate terrestrial data and reach areas not covered from the ground point of view (Figure 5). The actual cost of mobile Lidar systems, radio interferences of the platforms, and data accuracy make integrating ground-based and drone-based 3D laser scanners less feasible. The integration between TLS and photogrammetry by RPAS, or only an image-based survey, can be more effective and easier to plan. In the first, the main problem is related to the different resolution, precision, and accuracy of range and image data, particularly evident in these specific industrial architectures, to obtain a homogeneous 3D data system. Besides, in all the photogrammetric approaches, the mismatch between similar features in the same images block (Figure 2), appearing in both the foreground and background of a scene, represents the main problem. An image masking activity can avoid or reduce this mismatch. Some examples in the literature show that it is possible to use semantic image segmentation to eliminate the effect of moving objects in images (Nooruddin et al., 2018) or using the relative camera orientation to find a reliable object segmentation to increase the accurate 3D reconstruction (Mohammed et al., 2019). However, these and similar works (Kaneko et al., 2018; Wan et al., 2019) address the problem of continuous artifacts, while the domain issue is related to discontinuous elements structured according to spatial grids. The recent advances in Deep Learning methods represent a cutting-edge approach to solving very long and repetitive process, producing high-quality outputs. An example applied to a particular grid structure is offered by Knyaz et al. (Knyaz et al. 2020), who propose a Deep Learning approach for image filtering, overcoming the limit of photogrammetric processing applied to radio and television towers.

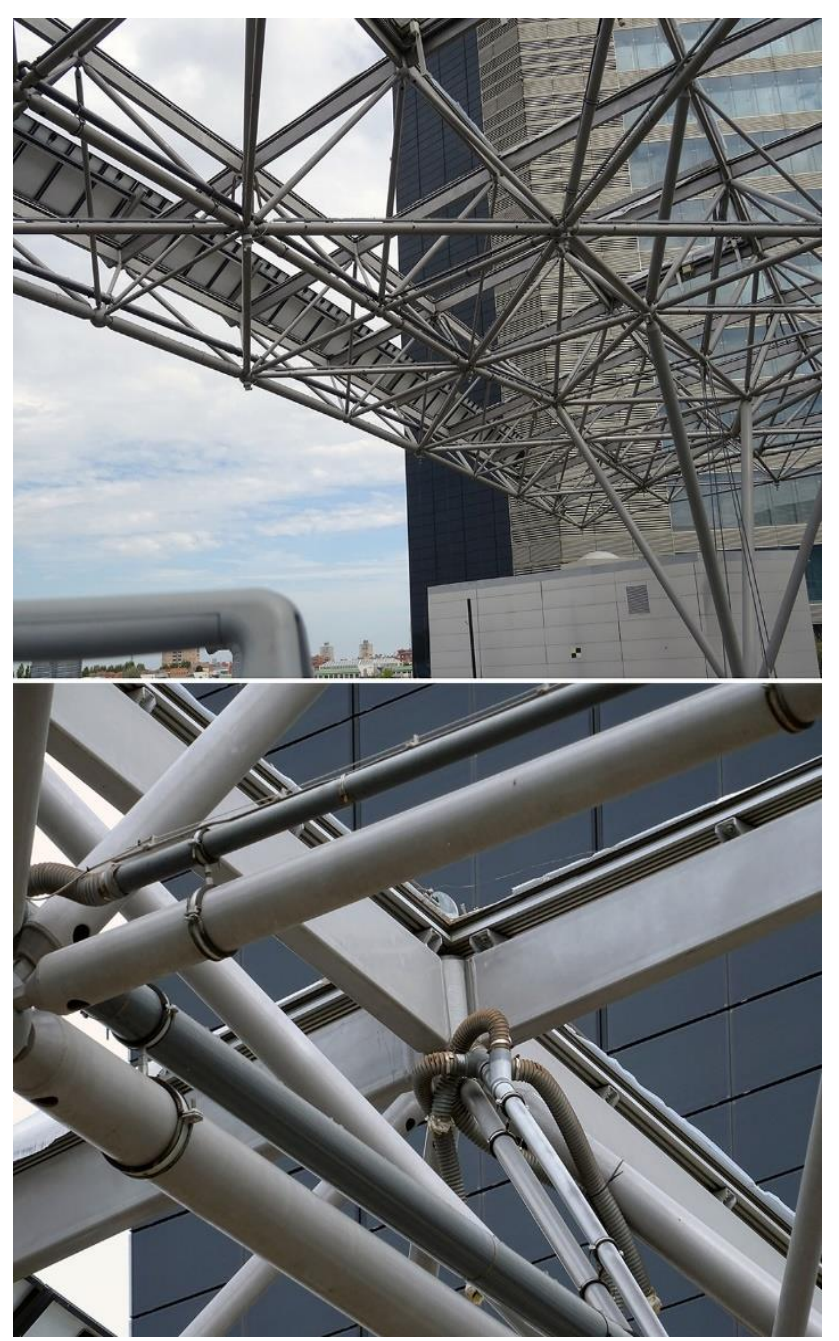

Figure 2: Example images of the mismatch problem due to nonuniform background (above) and the system installations with a similar size to the structural elements.

The paper proposes a reverse modeling approach, from the acquisition to the 3D modeling of a complex grid structure, not focusing on a specific aspect of the process but developing a complete path based on the integrated survey, the metrological verification, data processing, and $3 \mathrm{D}$ modeling. At the end of the process, pros and cons will be analyzed, foreshadowing future research on the topic. 


\section{THE CASE STUDY}

The paper discusses a steel space truss structure called "Vela", located in Bologna, Italy, and part of the architectural complex "Torre Unipol". The structure has a rhomboidal shape in projection, but a curved development in space, with two diagonal dimensions of 80 and 88 meters, a surface of $3600 \mathrm{~m} 2$, and a free span between the lateral supports of about $50 \mathrm{~m}$. The structure (Majowiecki and Berti, 2013) was built in 2012 with circular tubular profiles connected by spherical knots at the intrados and rectangular section tubular profiles connected by rigid knots flexibly at the extrados. The mesh of the extrados of the reticular is about $4 \times 4 \mathrm{~m}$. The cover was created with ETFE membrane cushions under pressure directly connected to the extrados profiles of the roof (Figure 3a). The architectural design was developed by Open Project Office and the structural project by Prof. Massimo Majowiecki and his Technical Office. An interdisciplinary team at DELFT accomplished the use of renewable energy and optimization of the cladding system.
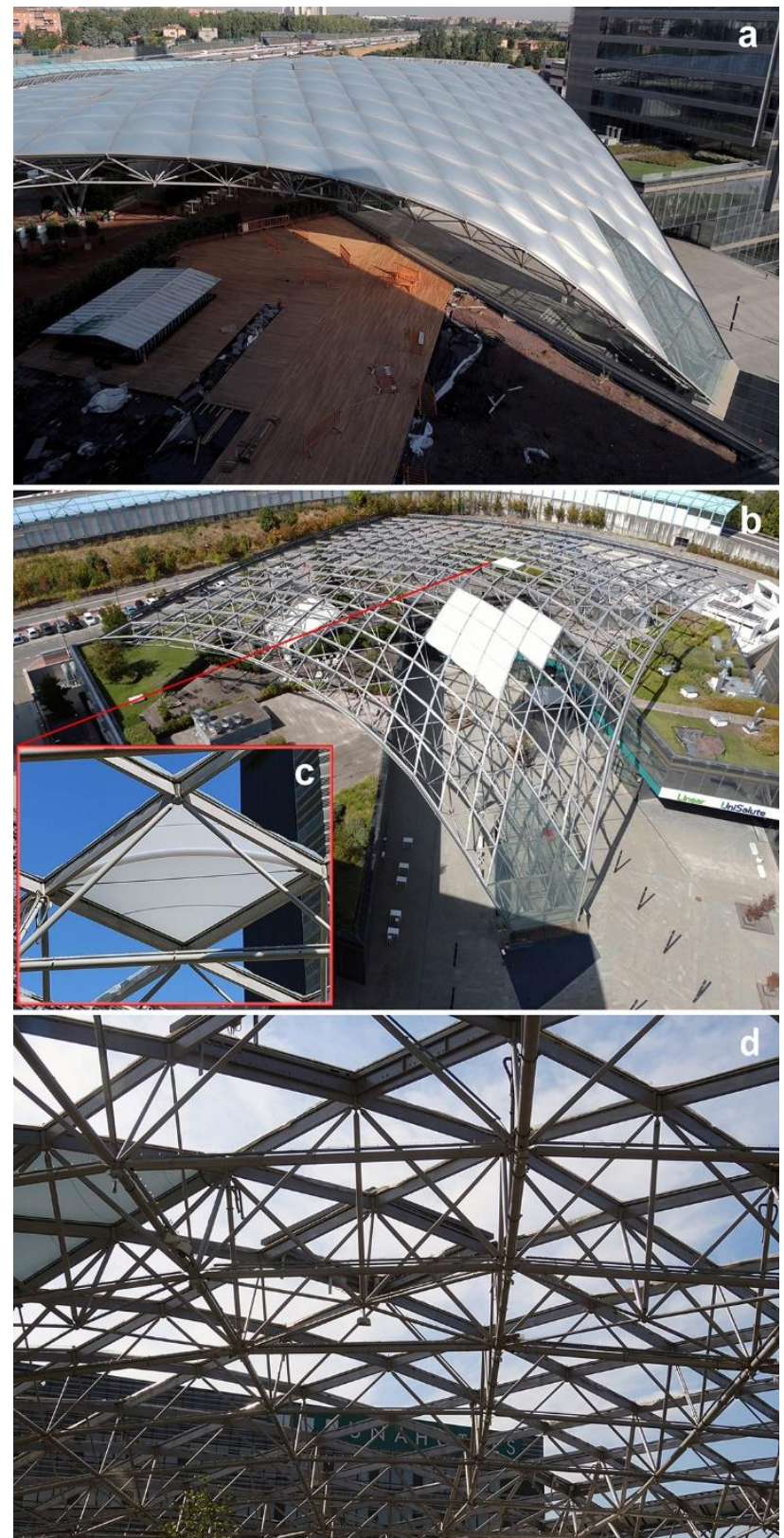

Figure 3: The 2012 space grid structure (a), the present one (b), a detail of the new cover (c), and an intrados view (d).
On June 22, 2019, the Emilia-Romagna region was hit by several thunderstorm cells, with large hailstorms, causing the rupture of all ETFE membranes of the Vela roof. After two years, in 2021, a project started to design a new covering system (Figure 3b, 3d). The roof intervention considers using an arch and a tie-rod placed in the diagonal of each mesh, on which a semi-transparent rigid cover is rested (Figure 3c). Every new rigid element must fit perfectly into the 253 meshes, defined by different shapes, sizes, and space positions.

A 3D survey of the structure was planned to create a 3D model, helpful in extracting all the meshes' principal dimensions for new component production. In detail, two diagonals of each extrados of the 253 meshes have been inspected (Figure 4), getting the dimensions from the external center and the axis of the extrados nodes. A maximum error of $2 \mathrm{~cm}$ in the relative position of each node was tolerated in the measurement accuracy. The purpose of the $3 \mathrm{D}$ acquisition is to create an accurate and reliable $3 \mathrm{D}$ point cloud for verifying the possible discrepancies between the asbuilt and the existing project, planning the technological interventions campaign.

\section{DATA ACQUISITION AND COMPARISON}

This steel space truss structure presents several survey bottlenecks. From the geometrical point of view, the roof develops according to curved generators with variable radii, becoming even more complex to predict and reduce the shadow effect given by installations (Figure 2) and structural elements (Figure 3). A second topic regards roof accessibility, intervisibility (Figure 5), and dimension. The height from the ground (up to $20 \mathrm{mt}$ ) excludes a direct survey. In addition, the top-down intervisibility defines a non-clear distinction between intrados and extrados areas. If both sides must be acquired, an RPAS use must be integrated with a terrestrial survey.

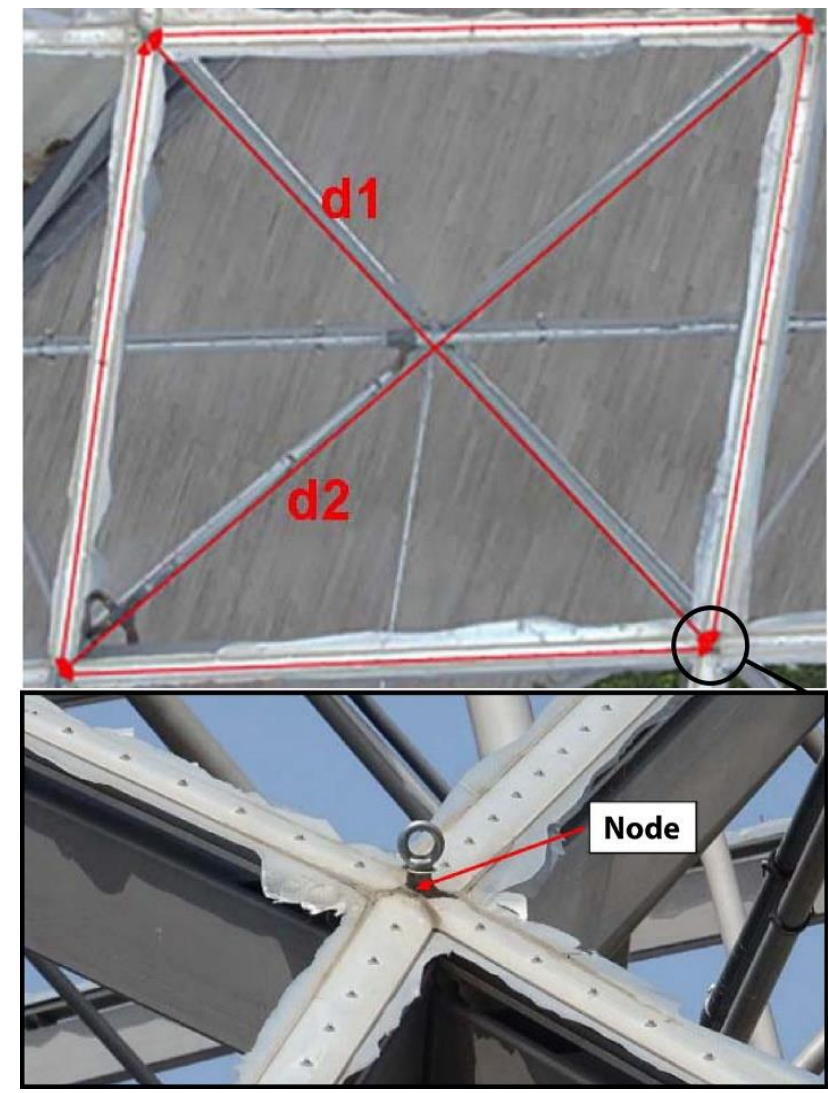

Figure 4: Diagonal extrados measures required for each mesh and detail of the node. 


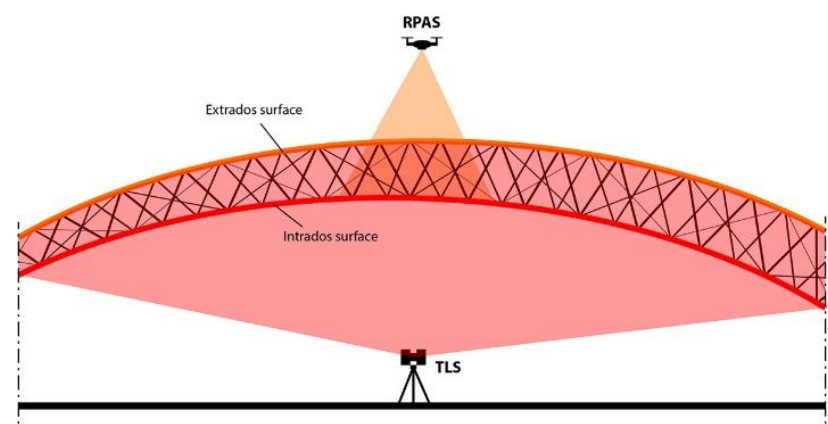

Figure 5: Intervisibility scheme between TLS and RPAS.

Some project prerequisites have influenced the operative step. The in-situ survey planning required the optimization of 3D scans to reduce people interferences and stress the relationship between working distance, individual components dimension, and data accuracy. Besides, the required precision and the involvement of ultralight RPAS, applied for safety reasons, excluded the RTK positioning system. At last, some tests about the optical instrument response on the metal structure were planned, verifying the noise, which may influence the data quality. An integrated survey approach based on 3D laser scanning, miniUAV photogrammetry, and topographic survey has been proposed to minimize these bottlenecks.

\subsection{Topographic framework}

In the GPCs distribution (Figure 6), to support both image-based and range-based orientation, the roof's inaccessibility played a key role, which obliged to locate GCPs on the main terrace below the structure. The 3D reference system of the whole survey is defined by three vertices, materialized on the wooden floor, and acquired by a total station. Four fixed targets were placed in the scene (T1, T2, T3, T4), while ten mobile aluminum targets were positioned ad hoc to orient images for each flight in the same XYZ system. For each of the three stations (S1, S2, S3), fixed and mobile GCPs were surveyed.

\subsection{Image-based survey}

A first photogrammetric survey of the whole structure has been planned to create a graphical representation of the extrados. The RPAS used was a DJI Mavic mini 2, equipped with a camera set up of $4 \mathrm{~mm}$ of focal length, f/2.8, ISO 100 , and $1 / 1000 \mathrm{sec}$ of exposition. The flying distance was 60 meters from the ground, with a GSD of about $2 \mathrm{~cm}$. The network vertices and the four fixed GPCs were used to orient a block of 35 images, processed in Agisoft Metashape SW, obtaining the roof's first 3D points cloud and orthoimage. The latter was helpful to plan the detailed photogrammetric campaign, dividing the structure into nine quadrants from $5 \times 5$ to $6 \times 6$ square meshes.

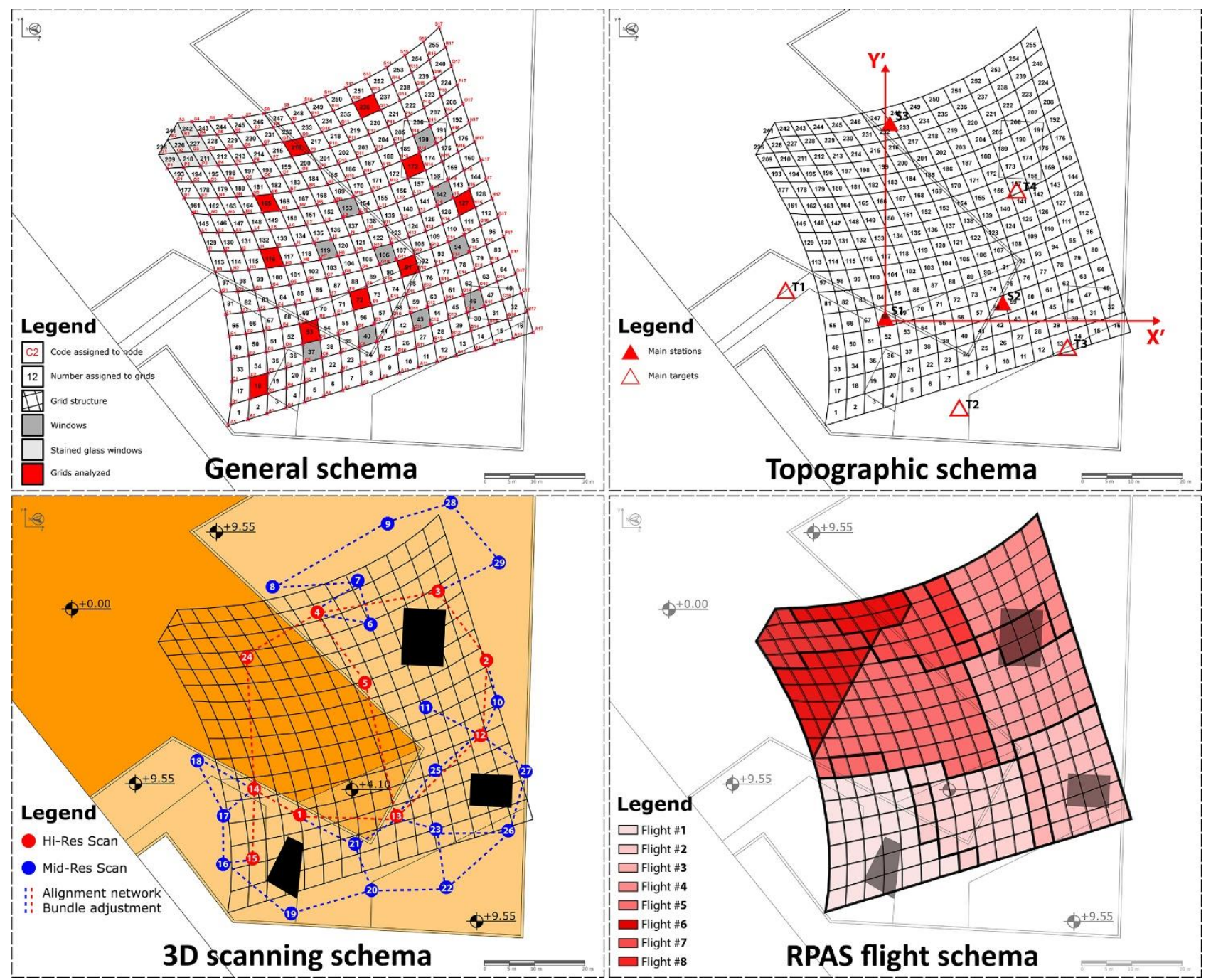

Figure 6: Structure schema, with its principal nomenclature and operational schemas of the survey campaign. 
The camera setup was repeated with a 6-7 meters flight altitude to the roof surface and a GSD of $2 \mathrm{~mm}$. Eight separate flights were done, preserving a longitudinal and lateral coverage of about $80 \%$ and reducing the image processing step to a maximum of 250 images for each block (Figure 6). The variation of the structure in altitude and inclination led to consider both oblique and nadiral images, guaranteeing adequate coverage and precision. A total of 1184 digital images were acquired and oriented using GPCs, determining the coordinates of the nodes. Each node coincides with the point of intersection of the axes of the steel plates (Figure 4). The presence of 10 windows on the roof (Figure 6) hid the nodes of the underlying mesh, obliging to cover two borders of the structure, obscuring the nodes in several images and limiting the number of usable ones. The coordinates were finally validated and collected in a list, calculating the six skewed distances (4 sides and two diagonals) that characterize each mesh.

\subsection{Range-based survey}

The 3D laser scanning started evaluating a uniform distribution of the scans for each quadrant and readapting a theoretical schema to the context (Figure 6). In the center, high-res scans (3 mm@10mt) fix the vertices of a primary alignment network. Many low-res scans (6 mm@10mt) provide a second-level network, reducing as much as possible the shadow areas. The Faro Focus S70 has been used for this specific application, and 29 scans were located considering the instrument intervisibility, optimizing the number of scans and the acquisition time. Staring for the reference scan (\# 15), oriented concerning the GPCs, all scans have been aligned using ICP and bundle adjustment processes. The low-res scans were filtered by distance, reducing the number of outlier data, and manually cleaned, refining the data quality. This operation took much time for the presence of many tangential acquisition effects. The clouds were then merged and subsampled at $5 \mathrm{~cm}$ resolution (Figure 7), regularizing the points distribution and optimizing the cloud management. survey the correspondent points in the intrados. Solar panels

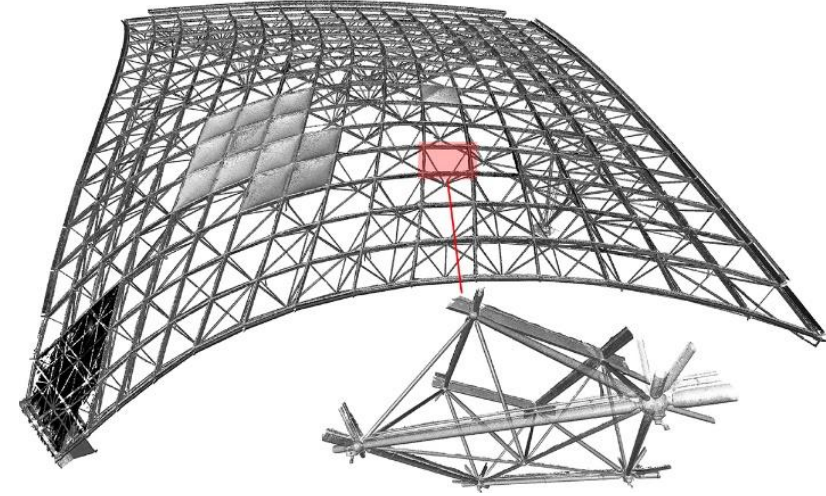

Figure 7: Range-based point cloud.

\subsection{Data integration and comparison}

The integration between the image-based and range-based data was carried out using common GCPs. The point cloud obtained from the 3D laser scanning defines a gold standard model, helpful to inspect the photogrammetric output locally (nodes distances) and globally (mesh comparison).

The barycentres of 20 nodes were extracted from the range-based point cloud, slicing specific portions of the nodes and fitting circular geometries. The $\mathrm{x}, \mathrm{y}$ coordinates of the center projected on the construction plane were used to calculate the main six distances of the mesh. The comparison with the photogrammetric node distances showed an average between $1 \mathrm{~mm}$ and a standard deviation of $2 \mathrm{~cm}$.

Besides, a careful masking activity related to the flight \#4 block has been carried out, reducing the noise and the spurious points in the photogrammetric cloud. Both range and image-based data have been superimposed and compared in CloudCompare software, showing an average deviation of $2.3 \mathrm{~cm}$ and an Std. Dev. of $3.1 \mathrm{~cm}$ (Figure 8). Due to the low data overlap between intrados and extrados, the values rise in some areas. The global comparison highlights a homogeneous behavior and the high accuracy of the photogrammetric cloud, excluding errors in $3 \mathrm{D}$ data orientation and construction.

\section{Point cloud comparison}
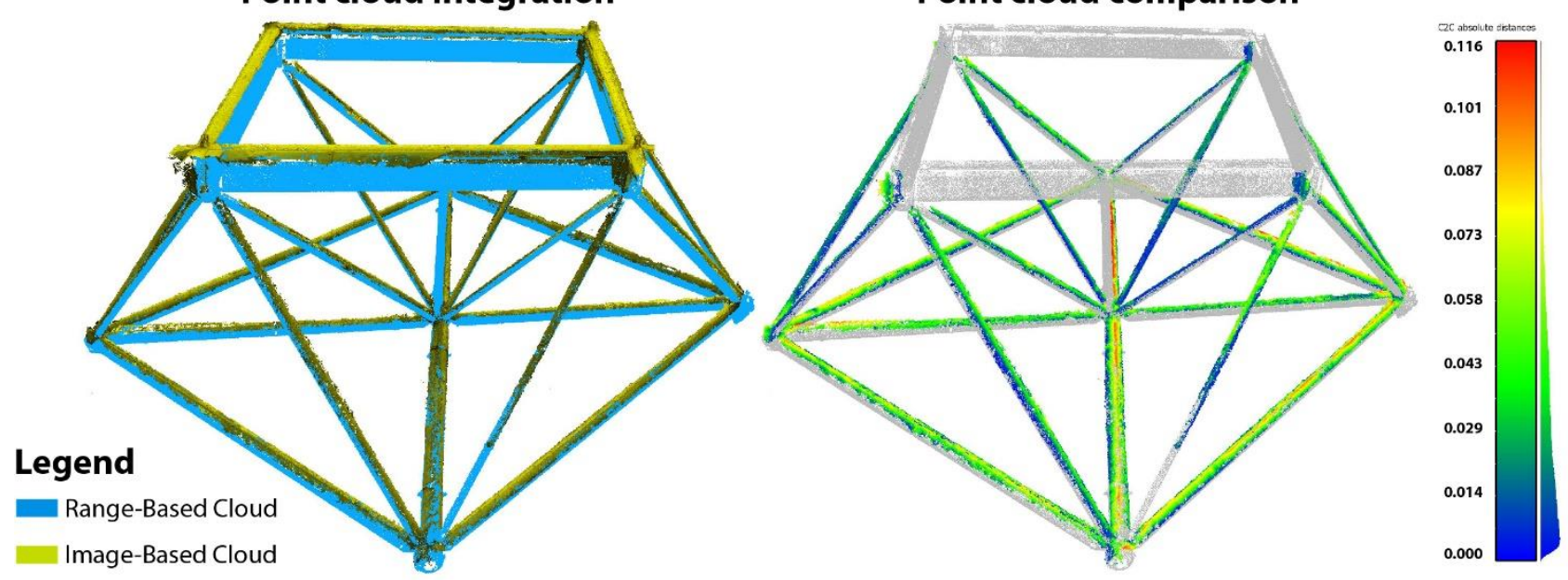

Figure 8: Integration between range-based and image-based point clouds and metric comparison in overlapping areas.

\section{DATA ANALYSIS AND MODELING}

The last passage in the 3D data process regards the point cloud translation into a parametric model. It is a challenging issue, mainly due to the accuracy of the starting $3 \mathrm{D}$ data and the construction of correct components in the space. Besides, the surface refinement of the industrial components reduces the interpretive chances compared to handmade artifacts. The proposed reverse modeling process focuses on mesh \#127, chosen for a complexity that exemplifies the entire structure. The executive project has been used at the end of the process to validate the experiment. 

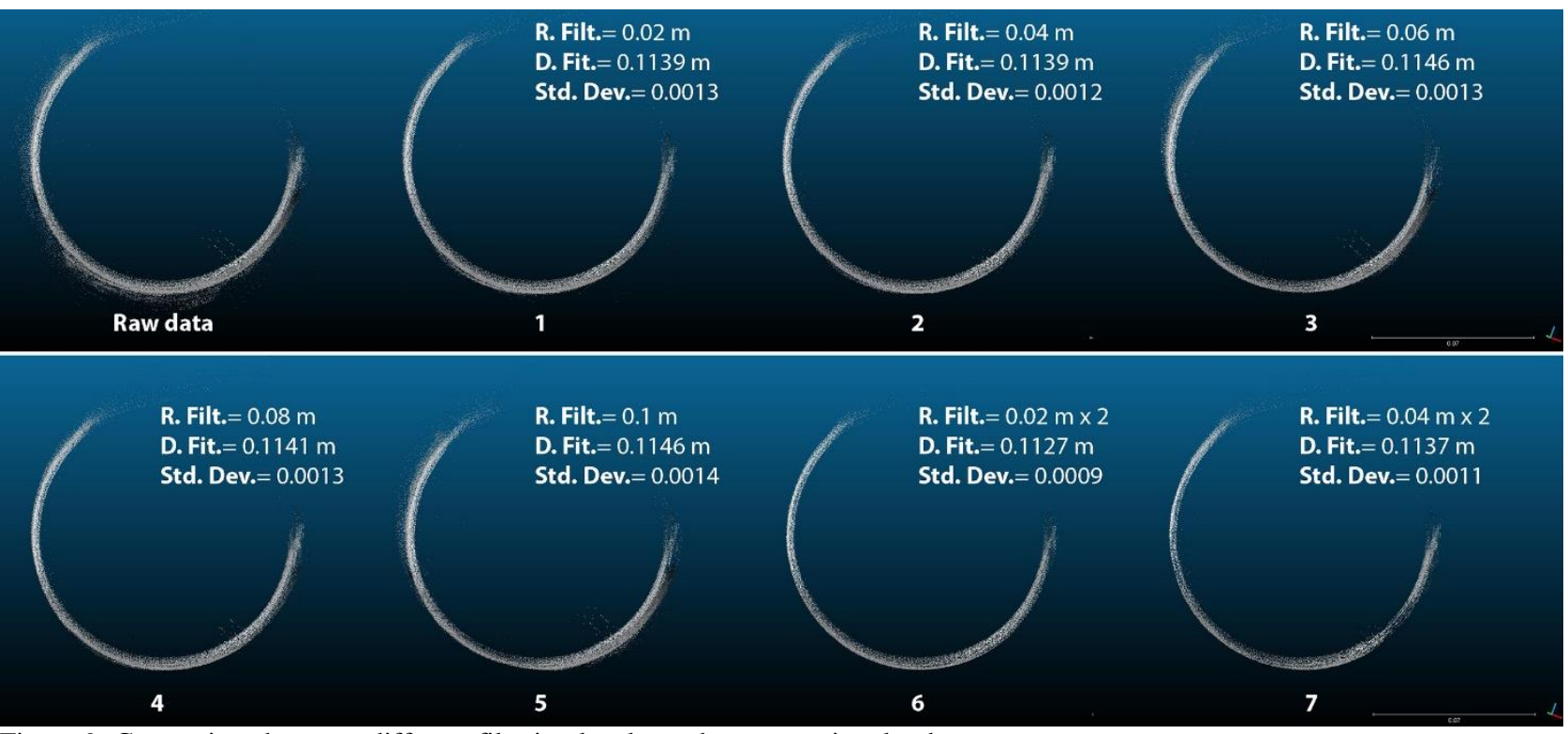

Figure 9: Comparison between different filtering levels on the same point cloud.

\subsection{Noise filtering}

Point cloud accuracy plays a crucial role in the 3D parametric reconstruction of regular shapes as spheres, cylinders, parallelepipeds. The quality in the 3D acquisition and alignment process defines the range of uncertainty in the geometrical construction. A cylindrical component of mesh \#127 was analyzed to test this passage. (Figure 9). Different levels of manual cleaning and automatic filtering were applied. A cylinder was fitted to the 3D point cloud for each level, comparing the obtained diameters and axes to the project values.

The experiment highlighted that variable filtering algorithms with different radii and iterations do not change the definition of the correct fitting geometry, showing the $+/-1 \mathrm{~mm}$ variation in diameter extraction. Instead, a filtering reiteration allows lowering the Std. Dev., making the positioning of the shape in the space more reliable, identifying the correct axis of the cylinder. Since automatic filters can clear most outliers, the optimal procedure is defined by mixing automatic and manual cleaning procedures.

\subsection{Features extraction}

Once the optimal cleaning setup was defined, it was applied to the entire mesh \#127, and the fitting process was repeated for all cylindrical components (\#20), spherical nodes (\#5), cylindrical nodes (\#4), and rectangular section extrados struts (\#4). For the latter, the plane fitting method was adopted. This operation has allowed identifying the main diameters, edges, sphere centers, and cylindrical axes of the geometry of the existing mesh, with a sub-millimetric average and Std. Deviation.

\subsection{Data classification and modeling}

Ten meshes distributed within the whole structure (Figure 6) were identified to ensure the most significant variability of structural components. The shape fitting process was applied for each one, extracting the main parameters such as radii, \#fitting points, mean, maximum and minimum distance, and standard deviation from the point cloud.

The values of the diameters were then sorted, highlighting the recurrence of values in specific ranges and the clustering in 10 classes (Figure 10-11) 7 referred to the structural components (yellow rectangle in Figure 7) and 3 to the air system cables

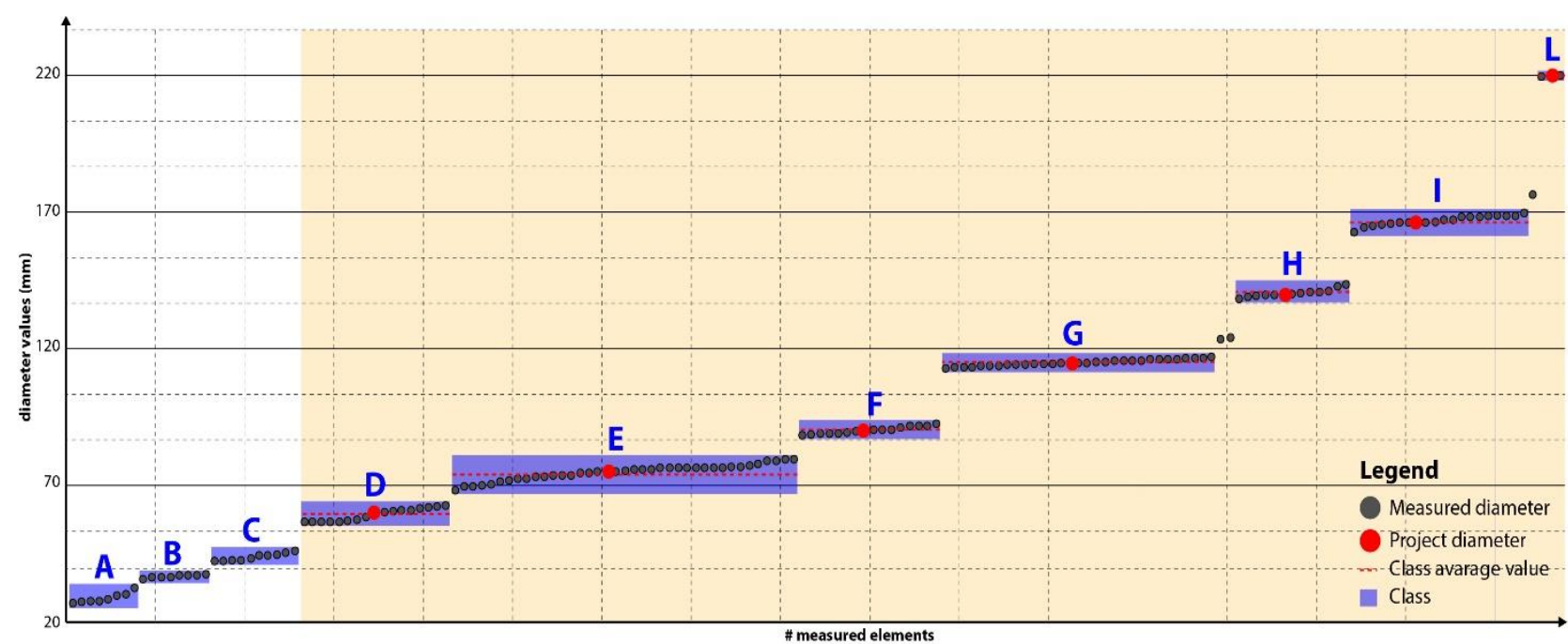

Figure 10: Distribution graph of detected samples. 


\begin{tabular}{|l|c|c|c|c|c|c|c|c|}
\hline Class & D & E & F & G & H & I & I* & L \\
\hline Project Name & D-3X & F-3X & G-1X & H-3X & M-3(X/Y) & N-1/7/8(X/Y/Z) & O-2(L/M/N) & P-8/12/14(X) \\
\hline Measure (mean) $(\mathrm{mm})$ & 58,8 & 74,4 & 89,7 & 114,6 & 140,0 & 166,5 & 166,5 & 219,4 \\
\hline Project diam. $(\mathrm{mm})$ & 60,3 & 76,1 & 88,9 & 114,3 & 139,7 & 165,1 & 168,3 & 219,1 \\
\hline Difference (mm) & $\mathbf{- 1 , 5}$ & $\mathbf{- 1 , 7}$ & $\mathbf{0 , 8}$ & $\mathbf{0 , 3}$ & $\mathbf{0 , 3}$ & $\mathbf{1 , 4}$ & $\mathbf{- 1 , 8}$ & $\mathbf{0 , 3}$ \\
\hline Std.Dev. & $\mathbf{2 , 4}$ & $\mathbf{2 , 8}$ & $\mathbf{1 , 3}$ & $\mathbf{1 , 2}$ & $\mathbf{1 , 4}$ & $\mathbf{1 , 7}$ & $\mathbf{1 , 7}$ & $\mathbf{0 , 6}$ \\
\hline
\end{tabular}

Table 1: Table comparing surveyed data to project data.
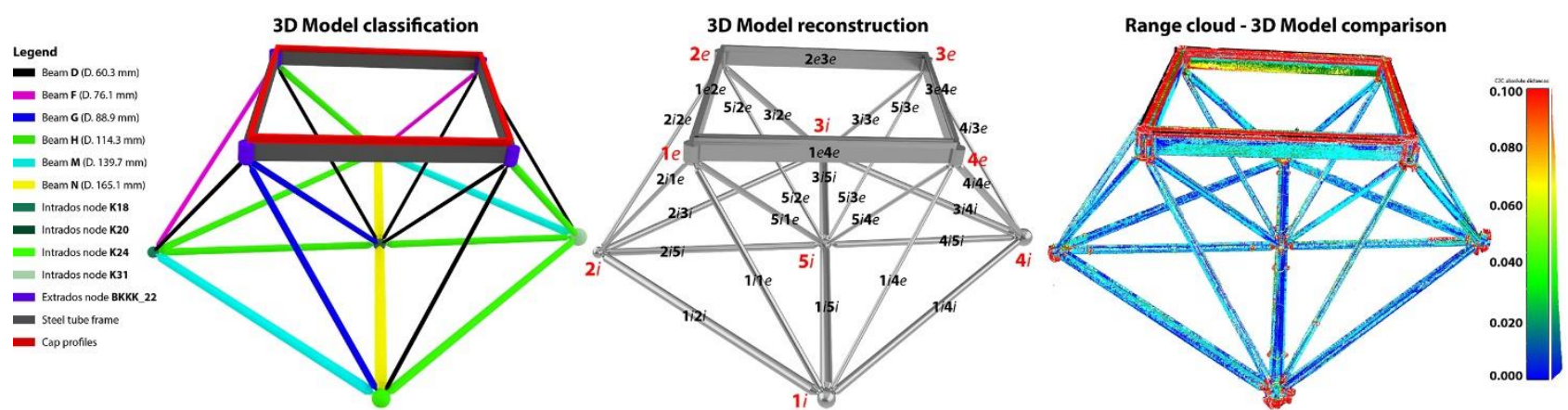

Figure 11: Classification scheme of mesh \#127, the 3D model with proposed nomenclature of the elements and comparison between the reconstructed 3D model and the surveyed one (range-based).

Thanks to the project data comparison, these latter have been identified, demonstrating the mismatch problem in identifying similar elements. One class (I) corresponds to two different project classes, very similar in dimension $(3 \mathrm{~mm}$ of diameter variation), so this approach could not distinguish the differences. The average of each class defined a priori as the most probable value for reconstructing every shape typology, reducing the discrepancy given by the direct interpretation. The values of the extracted averages for each class were compared with the project data, highlighting a millimetric difference (Table 1) and validating the process, except minimum variations. The validation process had a crucial role in generalizing the approach since project data may be unavailable. Besides, the experimental process forced to solve the nomenclature problem of the components, to identify each element within a large and complex structure. Based on the validated extracted values, mesh \#127 was reshaped (Figure 11). The diameter corrections were applied, preserving the previous cylindrical axes and generating the final shape.

\section{RESULTS}

The shown path can be applied to every space grid structure, even if unavailable project data. It allows identifying a sequence of classes of constructive components, improving the direct interpretation on the local 3D cloud, and standardizing the 3D construction of industrial elements. The final 3D model represents a reliable output from integrating range and imagebased data, local and global validation, filtering, features extraction, and classification. The project data has allowed validating the experimental process, which is very timeconsuming. The repetition 252 times for the whole structure (Figure 12) shows a crucial limit in applying the process to widescale, searching for future solutions that can automate some passages.

\section{CONCLUSIONS}

An integrated 3D survey approach has allowed overcoming some typical acquisition bottlenecks of the space grid structures. Comparing range-based and image-based data validates the process within the tolerances required to produce integrative components for the new roof cover. The manual point cloud classification and modeling, on the opposite, showed a reliable but time-consuming process. This critical aspect leads to possible future scenarios. The supervised Machine Learning approach may identify and classify recurring elements, reducing the time spent on a manual classification to support the scan-to-BIM process. Using automatic 3D shapes reconstruction based on computational modeling applied to classified points would be ground-breaking to solve the $3 \mathrm{D}$ reconstruction of complex space grid structures.

\section{ACKNOWLEDGEMENTS}

The authors would like to thank for their support and role in the project Eng. Giovanni Berti of MJW Structures, Prof. Massimo Majowiecki and Eng. Romano Piolanti of Open Project Srl.

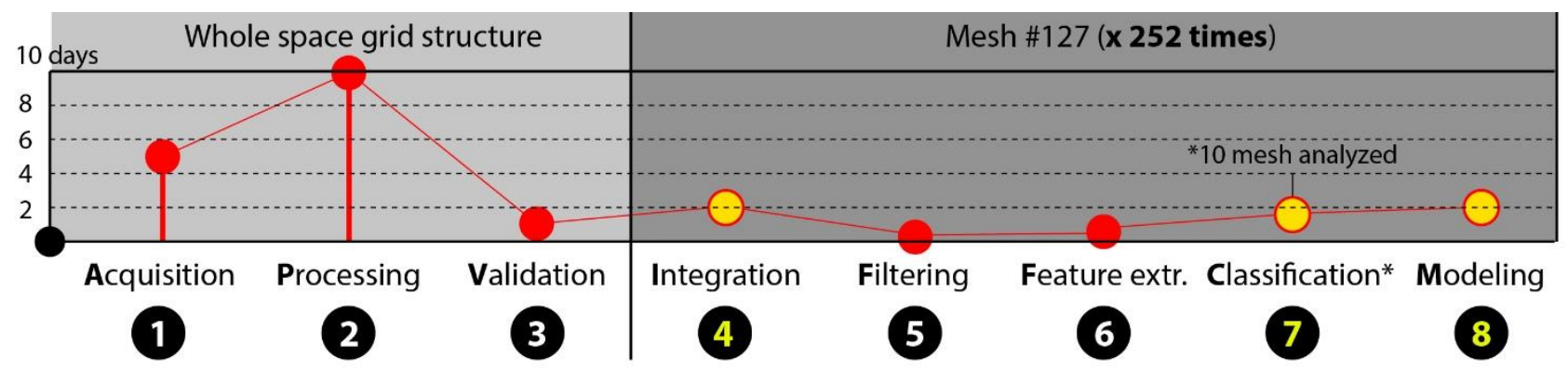

Figure 12: Timing for the entire structure and mesh \#127 processing. In yellow, the critical passages to extend overall the structure. 


\section{REFERENCES}

Abergel, V., Jacquot, K., De Luca, L.; Veron, P., 2021. Combining on-site and off-site analysis: towards a new paradigm for cultural heritage surveys. DisegnareCON, 14 (26), 2021, pp. $1-14$.

Adamopoulos, E.; Rinaudo, F., 2019. 3D interpretation and fusion of multidisciplinary data for heritage science: a review. Int. Arch. Photogramm. Remote Sens. Spatial Inf. Sci., XLII2/W15, pp. 17-24.

Bacharidis, K.; Sarri, F.; Ragia, L., 2020. 3D Building Façade Reconstruction Using Deep Learning. ISPRS Int. J. Geo Inf., 9, 322 .

Candela, G., Barrile, V., Demartino, C. and Monti, G., 2019. Image-based $3 \mathrm{~d}$ reconstruction of a glubam-steel spatial truss structure using mini-UAV. In Xiao, Y., Li, Z., \& Liu, K.W. (Eds.). (2019). Modern Engineered Bamboo Structures: Proceedings of the Third International Conference on Modern Bamboo Structures (ICBS 2018), June 25-27, 2018, Beijing, China (1st ed.). CRC Press.

Chilton, J., 2000. Space Grid Structures. Architectural Press, NewYork

Guidi, G., Remondino, F., Russo, M., Menna, F., Rizzi, A. and Ercoli, S., 2009. A multi-resolution methodology for the 3D modeling of large and complex archaeological areas. Special Issue in International Journal of Architectural Computing (IJAC), pp. 39-55.

Huang, H.; Wu, S.; Cohen-Or, D.; Gong, M.; Zhang, H.; Li, G.; Chen, B., 2013. L1-Medial Skeleton of Point Cloud. ACM Trans. Graph., 32, pp. 65-1-65-8.

Kaneko, M.; Iwami, K.; Ogawa, T.; Yamasaki, T.; Aizawa, K. 2018. Mask-SLAM: Robust Feature-Based Monocular SLAM by Masking Using Semantic Segmentation. In Proceedings of the 2018 IEEE/CVF Conference on Computer Vision and Pattern Recognition Workshops (CVPRW), Salt Lake City, UT, USA, pp. 371-3718.

Knyaz, V. A.; Kniaz, V. V.; Remondino, F.; Zheltov, S. Y.; Gruen, A., 2020. 3D Reconstruction of a Complex Grid Structure Combining UAS Images and Deep Learning. Remote Sensing, 12, 19: 3128 .

Liu, L.; Ceylan, D.; Lin, C.; Wang, W.; Mitra, N.J., 2017. ImageBased Reconstruction of Wire Art. ACM Trans. Graph. 36, pp. $1-11$.

Liu, Y-F.; Liu, X-G.; Fan, J-S.; Spencer, B.F.; Wei, X.C.; Kong, S.Y.; Guo, X-H. 2019. Refined safety assessment of steel grid structures with crooked tubular members. Automation in Construction, 99, pp. 249-264.

Lo Brutto, M., Iuculano, E.; Lo Giudice, P., 2021. Integrating topographic, photogrammetric and laser scanning techniques for a Scan-To-Bim process. Int. Arch. Photogramm. Remote Sens. Spatial Inf. Sci., XLIII-B2-2021, pp. 883-890.

Majowiecki, M.; Berti, G., 2013. La copertura reticolare spaziale "Vela" sotto la Torre Unipol a Bologna. Costruzioni Metalliche (CM), 4, pp. 36-47.
Martin, T.; Montes, J.; Bazin, J.C.; Popa, T., 2014. TopologyAware Reconstruction of Thin Tubular Structures. In SIGGRAPH Asia 2014 Technical Briefs; Association for Computing Machinery: New York, NY, USA.

Mohammed, H.M.; El-Sheimy, N. 2019. Segmentation of image pairs for 3d reconstruction. ISPRS Int. Arch. Photogramm. Remote Sens. Spat. Inf. Sci., XLII-2/W16, pp. 175-180.

Morioka, K.; Ohtake, Y.; Suzuki, H., 2013. Reconstruction of Wire Structures from Scanned Point Clouds. In Bebis, G., Boyle, R., Parvin, B., Koracin, D., Li, B., Porikli, F., Zordan, V., Klosowski, J., Coquillart, S., Luo, X., et al. (Eds), Advances in Visual Computing;; Springer: Berlin/Heidelberg, Germany, 2013; pp. 427-436.

Nooruddin, M.; Rahman, M. 2018. Improved 3D Reconstruction for Images having Moving Object using Semantic Image Segmentation and Binary Masking. In Proceedings of the 2018 4th International Conference on Electrical Engineering and Information Communication Technology (iCEEiCT), Dhaka, Bangladesh, pp. 32-37.

Perfetti, L., Polari, C. and Fassi, F., 2018. Fisheye multi-camera system calibration for surveying narrow and complex architectures. Int. Arch. Photogramm. Remote Sens. Spatial Inf. Sci., XLII-2, pp. 877-883.

Pereira, Á., Cabaleiro, M., Conde, B.; Sánchez-Rodríguez, A., 2021. Automatic Identification and Geometrical Modeling of Steel Rivets of Historical Structures from Lidar Data. Remote Sensing, 13 (11): 2108.

Su, I.; Qin, Z.; Saraceno, T.; Krell, A.; Mühlethaler, R.; Bisshop, A.; Buehler, M.J., 2018. Imaging and analysis of a threedimensional spider web architecture. J. R. Soc. Interface, 15, 20180193.

Turrin, M., Stouffs, R., Sariyildiz, S., 2010. Parametric design of the Vela roof. A case study on performance-oriented exploration of design alternatives. Proceedings of ASCAAD 2010, Fez, Morocco, 19-21 October 2010.

Yang, L., Cheng, J.C.P.; Wang, Q., 2020. Semi-automated generation of parametric BIM for steel structures based on terrestrial laser scanning data. Automation in Construction, 112: 103037

Wan, Q.; Li, Y.; Cui, H.; Feng, Z. 2019. 3D-MaskGAN:Unsupervised Single-View 3D Object Reconstruction. In Proceedings of the 2019 6th International Conference on Behavioral, Economic and Socio-Cultural Computing (BESC), Beijing, China, pp. 1-6.

Wei, X.-C., Fan, J.-S., Liu, Y.-F., Zhang, J.-X., Liu, X.-G.; Kong, S.-Y., 2021. Automated inspection and monitoring of member deformation in grid structures. Comput Aided Civ Inf., 00, pp. 121 .

\section{APPENDIX}

The research is the result of fieldwork and post-processing by both authors. M.R. is responsible for writing chapters 1,2,4,5,6,7, V.R. for chapter 3 . 